\title{
Introduction au dossier du congrès SELF Toulouse 2017
}

«Présent et Futur de l'ergonomie : Répondre aux défis actuels et être acteurs des évolutions de demain »

Béatrice Barthe, Olivier Gonon et Catherine Delgoulet

\section{(2) OpenEdition}

Journals

Édition électronique

URL : http://journals.openedition.org/activites/4360

DOI : 10.4000 /activites.4360

ISSN : 1765-2723

Éditeur

ARPACT - Association Recherches et Pratiques sur les ACTivités

Référence électronique

Béatrice Barthe, Olivier Gonon et Catherine Delgoulet, «Introduction au dossier du congrès SELF

Toulouse 2017 », Activités [En ligne], 16-2 | 2019, mis en ligne le 15 octobre 2019, consulté le 24

septembre 2020. URL : http://journals.openedition.org/activites/4360 ; DOI : https://doi.org/10.4000/ activites. 4360

Ce document a été généré automatiquement le 24 septembre 2020.

\section{(†) $\odot$

Activités est mis à disposition selon les termes de la licence Creative Commons Attribution - Pas d'Utilisation Commerciale - Pas de Modification 4.0 International. 


\section{Introduction au dossier du congrès SELF Toulouse 2017}

«Présent et Futur de l'ergonomie : Répondre aux défis actuels et être acteurs des évolutions de demain »

Béatrice Barthe, Olivier Gonon et Catherine Delgoulet

1 Ce dossier fait suite au $52^{\mathrm{e}}$ congrès de la Société d'Ergonomie de Langue Française, intitulé «PRÉSENT et FUTUR de l'ergonomie: Répondre aux défis actuels et être acteurs des évolutions de demain ", qui s'est déroulé à Toulouse les 20-22 septembre 2017.

2 Pour reprendre les mots-clés de cet intitulé de congrès, au PRÉSENT l'ergonomie comme discipline scientifique et comme pratique - produit des connaissances sur l'activité des femmes et des hommes au travail, ainsi que sur les méthodes d'intervention et de mise en œuvre du changement. Ces connaissances, intégrées à la transformation et à la conception des situations et des organisations du travail, évoluent en même temps que les contextes sociaux et économiques: les nouvelles formes d'organisation du travail, le développement des services, les transformations technologiques ou règlementaires, l'évolution des populations au travail, etc. Ces mutations du travail participent au futur de l'ergonomie. Au-delà de ces évolutions, le FUTUR de l'ergonomie peut également être envisagé dans une visée anticipatrice. En ce sens, un positionnement proactif de l'ergonomie lui permet d'être davantage partie prenante de ces évolutions. Ainsi, l'ergonomie du futur peut par exemple se positionner en amont des réflexions autour des innovations notamment sociales, organisationnelles ou technologiques.

3 L'ambition de ce congrès était de favoriser les échanges et les débats sur un positionnement davantage proactif de l'ergonomie, thème général et fil rouge du congrès, tout en laissant la place à l'ensemble des pratiques et des recherches en ergonomie qui s'inscrivent dans les évolutions et les défis actuels.

4 Ce congrès s'est également inscrit dans le futur des congrès de la Société d'Ergonomie de Langue Française, en proposant des innovations au niveau de sa politique scientifique. Cela s'est traduit par une volonté d'ouverture sur la diversité des recherches et des pratiques en ergonomie. L'idée était de rassembler ergonomes et 
collaborateurs des ergonomes, de faire des congrès de la SELF un lieu ouvert, régulier, un rendez-vous annuel, en structurant le programme scientifique en thèmes, thèmes qui visent à courir d'une année sur l'autre et qui constituent ainsi un socle commun.

5 Ainsi, des dix thèmes définis dans la phase de conception des axes scientifiques $d u$ congrès qui structurent à présent les congrès de la SELF, six sont représentés dans ce dossier pour la revue Activités. Ils relèvent de la santé au travail et de la prévention des risques professionnels, de l'organisation du travail, de la fiabilité humaine et organisationnelle, de la contribution des ergonomes au champ de l'innovation technologique, de la conception et l'animation de formations, et enfin, des recherches et pratiques sur l'intervention en ergonomie.

6 Sept contributions présentées ci-après développent plus particulièrement un de ces thèmes au prisme de formes diverses d'analyse de l'activité.

- Le texte de Maxime Norval, Mohsen Zare, René Brunet, Fabien Coutarel et Yves Roquelaure, intitulé «Intérêt de la Marge de Manœuvre Situationnelle pour le ciblage des situations à risque de Troubles Musculo-Squelettiques ", constitue une illustration du thème de la Santé au travail et des risques professionnels. Il rend compte d'un travail réalisé dans une grande entreprise du secteur de la métallurgie sur l'évaluation d'une démarche originale, mobilisant le concept de «marge de manœuvre situationnelle » (MMS), pour le ciblage des situations de travail potentiellement à risque au regard des enjeux de prévention des Troubles Musculo-Squelettiques.

- La thématique Organisation du travail englobe tout autant les questions du temps de travail, que celles liées au management ou plus généralement au collectif dans les situations de travail. Ici, la contribution de Valérie Zara-Meylan et Serge Volkoff, «Gérer les temps pour gérer la qualité : l'activité de traitement des dossiers de retraite», examine comment l'activité de gestion temporelle de techniciens en charge du traitement des dossiers retraite s'enrichit avec l'expérience et constitue un gage de qualité de la prestation de service. L'objectif de cette recherche-action est d'identifier les besoins et enjeux de cette activité, ainsi que les configurations qui permettent la gestion des dilemmes temporels ou au contraire la rendent difficile.

- Le texte de Fabrice Bourgeois, Christophe Vanpoulle et Christine Ammirati, «Dans la conception des salles de régulation du SAMU, pouvoir communiquer est un enjeu de fiabilité de ses missions", met l'accent sur les enjeux de Fiabilité humaine et organisationnelle, troisième thème du congrès de la SELF représenté dans ce dossier, dans un centre d'appels d'un service de médecine d'urgence. Les auteurs soulignent comment l'activité de communication au centre du travail de régulation des appels, permet d'éviter des ruptures de flux dans le traitement des appels, des attentes problématiques et, en conséquence, à viser une plus grande réactivité et fiabilité du service rendu.

- La contribution des ergonomes au thème Innovations technologiques, organisationnelles, sociales, est illustrée par le texte d'Émilie Loup-Escande et Jean-Marie Burkhardt : «Évaluer l'utilité dans le contexte des technologies émergentes pour identifier des besoins latents : éléments issus d'une analyse des interactions en situation d'usage». Cette contribution vise à comprendre dans quelle mesure l'évaluation d'une technologie émergente, en contexte écologique et au moyen d'un prototype, favorise la génération de besoins latents. Ces besoins ne sont pas identifiés a priori ; ils émergent d'interactions entre les participants dans des situations de formation et de travail.

- Deux contributions étayent les questionnements qui alimentent le thème Ergonomie Formation et Apprentissage. Proposant « Un cadre interprétatif pour enrichir la réflexivité : le 
cas d'une formation à la médiation civile et commerciale ", Valérie Saint-Dizier de Almeida, Flora Ilardo, Isabelle Serre, Olivier Cachard et Guy Deloffre abordent, auprès d'une population d'avocats et de notaires, l'interrogation des pratiques professionnelles par les professionnels eux-mêmes en discussion collective à partir d'un jeu de rôle. Ancré dans une approche issue des sciences du langage et de la psychologie de la communication, l'enjeu est de proposer de nouveaux cadres interprétatifs afin que les professionnels perçoivent leur activité sous de nouveaux angles et que leurs pratiques en soient enrichies.

- Dans un autre registre, l'article de Stéphanie Shot, Simon Flandin, Annie Goudeaux, Laurence Seferdjeli et Germain Poizat, «Formation basée sur la perturbation : preuve de concept par la conception et le test d'un environnement numérique de formation en radiologie médicale", analyse le potentiel bénéfice des perturbations en situation d'apprentissage. Les auteurs évaluent en quoi l'environnement numérique conçu permet d'outiller les formateurs d'autres modalités formatives qui ne reposent pas uniquement sur la transmission de savoirs établis, mais visent à produire un «choc " pour encourager la production de nouvelles significations et le développement de nouvelles modalités d'action et d'organisation.

- Enfin, la contribution de Yann Poley et Johan Petit, «Comment contribuer au développement des pratiques des représentants du personnel dans les CHSCT : le cas d'une recherche-intervention à la SNCF ", relève d'une réflexion sur les Recherches et les pratiques de l'intervention en ergonomie, dernier thème représenté dans ce dossier. Cet article apporte un éclairage sur l'activité des représentants du personnel (CHSCT), dans une perspective de développement de leur capacité à traiter les questions du travail, c'est-à-dire à penser, agir et débattre autrement du travail des salariés et également de leur propre travail. Dans ce cadre, les auteurs présentent les temps et les actions sur lesquels l'ergonome peut accompagner les représentants du personnel et, plus largement, les membres d'une Instance Représentative du Personnel. 